\title{
In vitro Comparison between Two Different Implant Titanium Surfaces in Osseointegration Process
}

\author{
Andrea Ballini, Apollonia Desiate, Stefania Cantore
}

\begin{abstract}
The osseointegration rate of titanium dental implants is related to their composition and surface roughness. Rough-surfaced implants favor both bone anchoring and biomechanical stability.

The future of dental implantology should aim to develop surfaces with controlled and standardized topography or chemistry.

This approach will be the only way to understand the interactions between proteins, cells and tissues and implant surfaces. The local release of bone stimulating or resorptive drugs in the peri-implant region may also respond to difficult clinical situations with poor bone quality and quantity, such as implant design and surface. These therapeutic strategies should ultimately enhance the osseointegration process of dental implants for their immediate loading and long-term success. Aim of this work was to compare implant titanium surfaces prepared with two different topographies for evaluating osteoblasts adhesion and growth.
\end{abstract}

Keywords: O steoblasts, Implant surfaces, Osseointegration, Titanium, Dental implants.

How to cite this article: Ballini A, Desiate A, Cantore S. In vitro Comparison between Two Different Implant Titanium Surfaces in Osseointegration Process. Int J Experiment Dent Sci 2012; 1(2):84-88.

\section{Source of support $\mathrm{Nil}$}

Conflict of interest: None

\section{INTRODUCTION}

A $n$ increasing number of dental and orthopedic implants of differing material, design and surface topography are inserted in humans every year. A high degree of clinical success has been reported for some dental implants such as the B ranemark system, ${ }^{1,2}$ whereas other oral implant systems have not been found very successful, ${ }^{3,4}$ and some systems regarded as promising are only supported by results from a few years of follow-up. ${ }^{5}$

Why some implant systems work well whereas others do not is not completely understood, even though knowledge is increasing about how implant surfaces interact with different tissues. Some properties of the biomaterials used, the host tissue and the surgical technique are considered to be more important than others to achieve successful implant incorporation in living tissue.

A lbrektsson et al ${ }^{6}$ proposed six factors which have been generally accepted as especially important for the establishment of reliable osseointegration: I mplant material, implant design, surface conditions, status of the bone, surgical technique and implant loading conditions.
Surface roughness is one property that contributes to the surface condition. Rougher surfaces have been shown to result in firmer bone fixation. ${ }^{7}$ H owever, knowledge about the optimal surface roughness has not been available, to part depending on a lack of appropriate measuring equipments for screw-shaped implants.

A im of this work was to compare implant titanium surfaces prepared with two different topographies for evaluating osteoblasts adhesion, and cell growth, with a protocol standardized form our previous experience. ${ }^{8}$

\section{MATERIALS AND METHODS}

\section{Patients}

Specimens of alveolar bone were obtained from three Caucasian patients, 20 to 30 years old, undergoing oral surgery, after informed consent. N one of them suffered from periodontal disease or was under medical care interfering with bone metabolism at the moment of investigation.

\section{Cell Culture}

Specimens obtained from alveolar wall, interdental septum or cortex of included tooth were mechanically cleaned and digested with $0.5 \mathrm{mg} / \mathrm{ml}$ collagenase at $37^{\circ} \mathrm{C}$ for 30 minutes to remove any soft tissue. Degradation products were removed through repeated washing with minimum essential media-al pha (M EM - $\alpha$ ) supplemented with $100 \mathrm{UI} / \mathrm{ml}$ penicillin, $100 \mathrm{mg} / \mathrm{ml}$ streptomycin, $50 \mathrm{UI} / \mathrm{ml}$ mycostatin and $2.5 \mathrm{mg} / \mathrm{ml}$ amphotericin B. Cleaned bone fragments were placed at the bottom of a $100 \mathrm{~mm}^{2}$ dish and grown in $\alpha$-MEM medium supplemented with $10 \%$ fetal calf serum (FCS) and antibiotics at $37^{\circ} \mathrm{C}$ in a humidified atmosphere of $5 \% \mathrm{CO}_{2}$. First medium replacement was done 4 days after starting cell culture and then it was replaced every 3 days. M icroscopic examinations were carried out daily to check the emergence of primary osteoblast-like cells (Figs $1 \mathrm{~A}$ and B). A fter 4 to 7 days first cells were visible and in 2 weeks they reached confluence.

\section{Cellular Phenotyping by Alkaline Phosphatase Staining}

Identification of the cellular population, as osteoblasts, was performed before starting all experiments. The functional staining for the alkaline phosphatase (ALP) activity was used as early marker of the osteoblastic character of emerged 

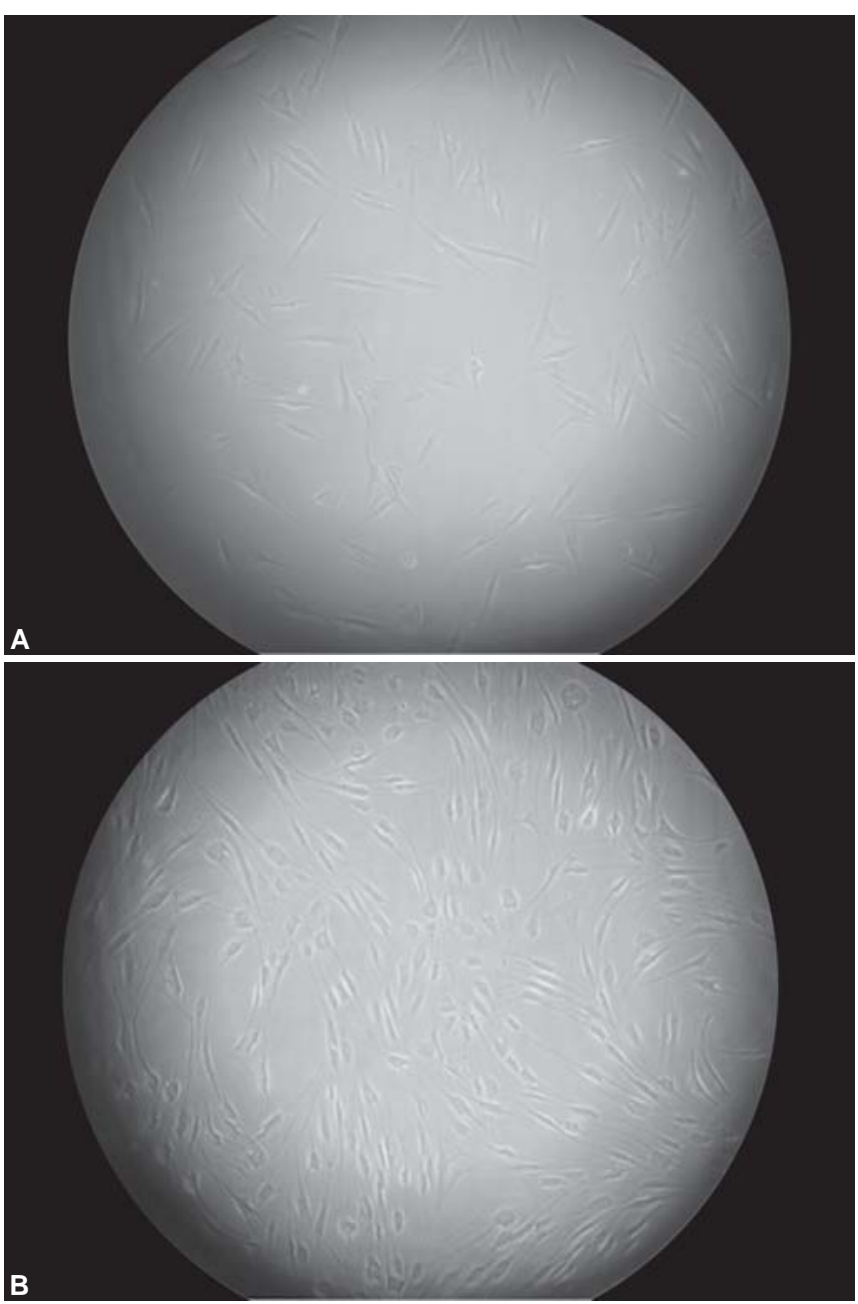

Figs $1 \mathrm{~A}$ and $\mathrm{B}$ : Osteoblasts observed at contrast phase microscopy after 1 day $(A)$ and 1 week (B)

cells before and after their growth in a differentiating medium. Emerged cells were pooled in one single suspension and seeded in 6-well plates at a density of 100,000 cells/plate. At confluence, they were induced to differentiate supplementing MEM growing medium with $50 \mu \mathrm{g} / \mathrm{ml}$ of freshly prepared ascorbic acid and $10^{-8} \mathrm{M}$ dexamethasone (DEX). The day of medium replacement was considered as day zero. A LP activity was evaluated at day zero and after 5 and 10 days of growth in the differentiation medium. At each time cells were fixed and incubated for 30 minutes in Tris buffer $0.2 \mathrm{M}$, pH 8.3 with A S-MX phosphate as a substrate and fast blue as a stain (Sigma, St L ouis, M O, USA ). The A LP-positive cells were blue/purple stained.

\section{Cellular Differentiation by ALP Activity}

Cells were grown in the same way as the phosphatase staining. At day zero and after 7 and 14 days, cells were fixed with a $2 \%$ paraformal dehyde solution and cellular membranes solubilized with a $0.1 \%$ sodium dodecyl-sulfate solution and tested for A LP activity (4-methylumbelliferyl phosphate disodium salt, Sigma). Briefly, cellular lysates were incubated at $37^{\circ} \mathrm{C}$ for 30 minutes in alkaline

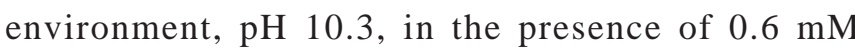
4-methylumbelliferyl phosphate as A LP substrate. The amount of the substrate converted to the fluorogenic product 4-methylumbelliferyl was proportional to the osteoblast A LP activity. 4-methylumbelliferyl phosphate fluorescence was read at $448 \mathrm{~nm}$ after excitation at $364 \mathrm{~nm}$. A rbitrary units of fluorescence were converted in $\mathrm{nmol} / \mathrm{min}$ by using calibration curves and total protein content measured with the BCA kit (Pierce). Finally ALP activity was expressed as $\mathrm{nmol} / \mathrm{min} / \mathrm{mg}$ protein.

\section{Synthesis of Nodes of Mineralized Matrix}

Osteoblasts were seeded in the same way as for cellular differentiation analysis. A total of $10 \mathrm{~mm} \beta$-glycerophosphate was added to the differentiation medium for 5 weeks to stimulate matrix synthesis and nodes of mineralization deposition. The osteogenic medium was changed twice a week and after 5 weeks of treatment, cells were stained according to $\mathrm{V}$ on $\mathrm{K}$ ossa protocol. ${ }^{9} \mathrm{~B}$ riefly cells were fixed in a solution of $2 \%$ paraformaldehyde, $2 \%$ sucrose for 10 minutes, washed 3 times with distilled water and stained with a $5 \%$ solution of $\mathrm{AgNO}_{3}$. The staining consisted of 30 minutes room temperature incubation in the dark and three washes with distilled water followed by 1 hour of exposition to artificial intense light. Pictures were acquired using a digital camera connected to A xiovert 200 inverted fluorescence microscope and analyzed.

\section{Cellular attachment and Growth on Titanium Surfaces}

Osteoblasts were seeded as for cellular differentiation analysis on titanium sterile surfaces laid at the bottom of 6-well plates (Fig. 2). The implant titanium surfaces were selectioned with two different topographies: 0 ne as machined surface used as control (CTRL - -a derivative from

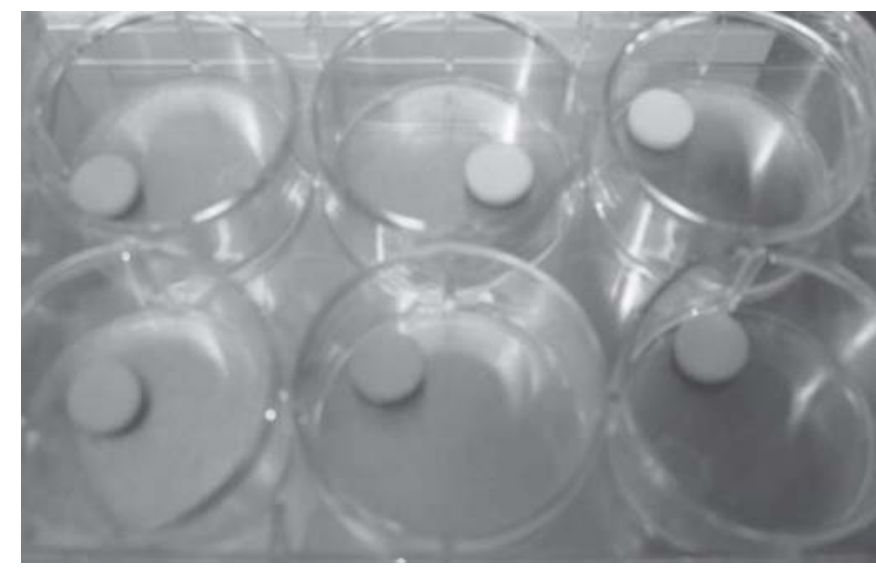

Fig. 2: Multiwell with titanium surfaces 
machined titanium implants, not branded) and one blasted surface as rough-surfaced (TEST-O ssean ${ }^{\circledR}$ Surface INTRA-LOCK). All implants were cleaned in an ultrasonical bath using trichlorethylene as detergent and rinsed two times in absolute ethanol.

At 24 hours after seeding nonadherent cells were washed out from the dish and the topography of the attached cells was investigated. For this purpose $10 \mu \mathrm{m}$ dichlorofluorescein diacetate (DCF-DA, Sigma) was added to the culture medium for 20 minutes at $37^{\circ} \mathrm{C}$ in the dark. The green fluorescence of DCF was analyzed at the confocal laser microscope (LSCM) by exciting samples with the A r-Kr laser beam at $507 \mathrm{~nm}$ and analyzing emission at $530 \mathrm{~nm}$. A cquisition, storage and analysis of data were made with LaserSharp and LaserPix BioR ad software. For calculation of cell growth curve on titanium surfaces, 100,000 osteoblasts/plate were seeded in the same way as for cellular differentiation analysis on a titanium surface laid at the bottom of 6 -well plates. Every 24 hours cells were detached and the cell number counted over a period of 5 days.

\section{RESULTS}

\section{ALP Activity}

Cells emerging from bone biopsy were phenotyped for their differentiating capacity by ALP staining before starting subsequent experiments.

Initial condition (time 0) consisted in replacement of normal medium with the differentiating medium. The functional ALP staining was repeated 5 and 10 days after medium replacement and irradiation and a stronger staining in all cells compared to time 0 was found (Fig. 3).

\section{Bone Mineral Matrix Nodules}

Cells at confluence were induced to synthesize bone mineral matrix nodules by replacing normal medium with an osteogenic medium. After 5 weeks matrix nodules were revealed by von Kossa staining both on machined and blasted implant surfaces (Figs 4 A and B ). B lasted surfaces were more efficient than controls in producing matrix nodules (Graph 1).

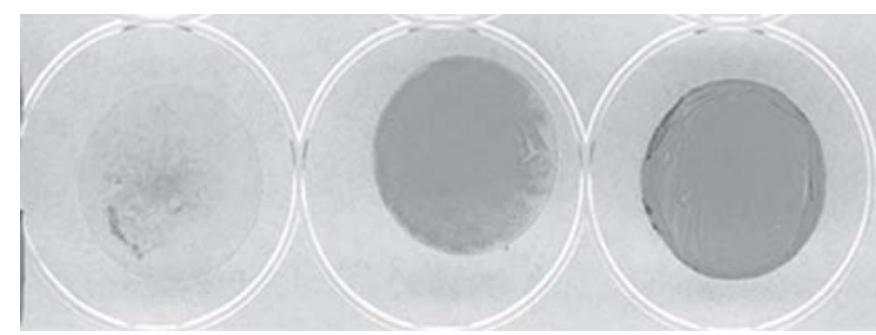

Fig. 3: Histochemical ALP at time 0 (T0), 5 days (T5) and 10 days $(\mathrm{T} 10)$
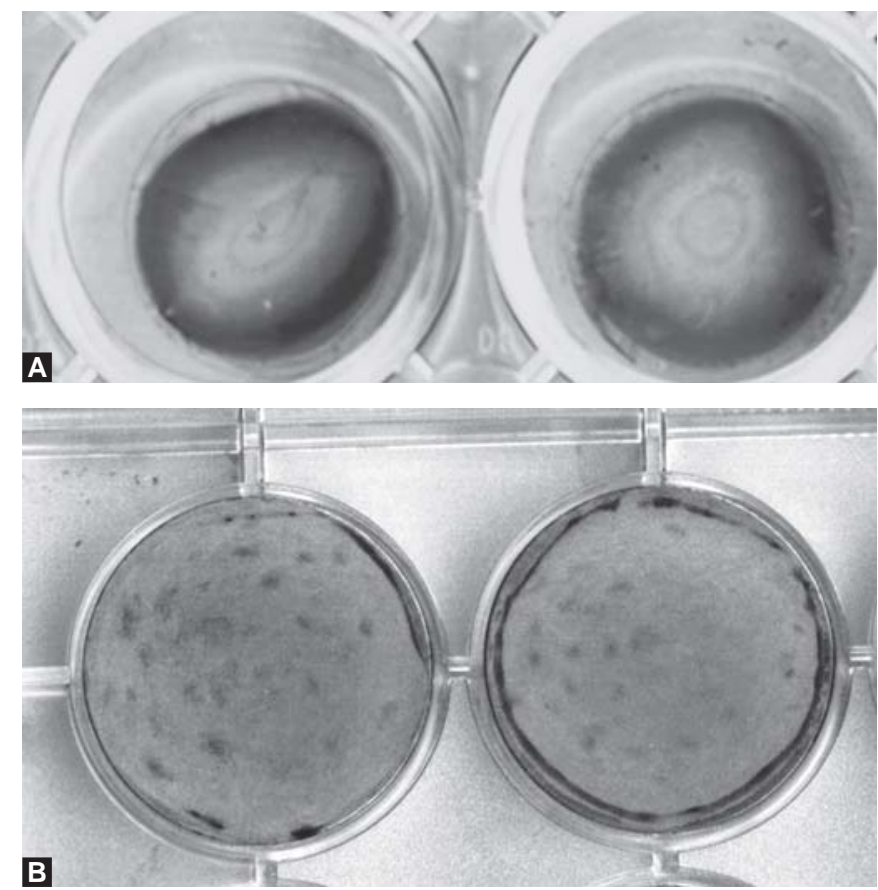

Figs 4A and B: Synthesis of mineralized matrix nodules (A) machined, (B) blasted

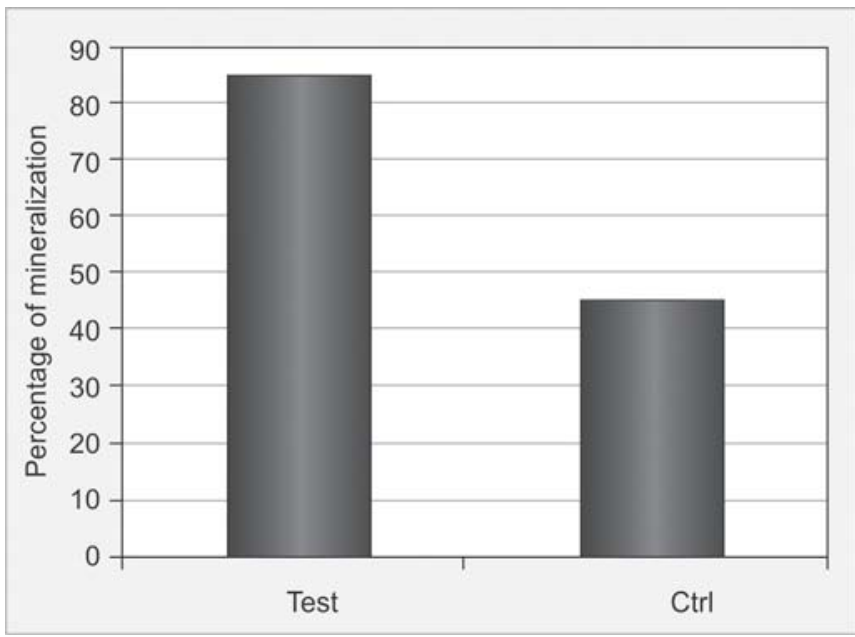

Graph 1: The quantitative analysis of mineralization kinetic was carried out by scanning the mineralized areas by image analyzer. The values represented in the graph are expressed as a percentage of the total mineralized surface relative to the well

\section{Cellular Profile on Titanium Surfaces}

The degree of cellular attachment osteoblasts to implant surfaces was assessed by confocal laser scanning microscope. Cell cultures areas were labeled by fluorescent dichlorofluorescein diacetate, which detects level of reactive oxygen species (ROS) production. Values are expressed as a percentage of the area occupied by cells compared with the total area of surface (Figs $5 A$ and $B$ ). The data show that cells were preferably attached on blasted surface. 

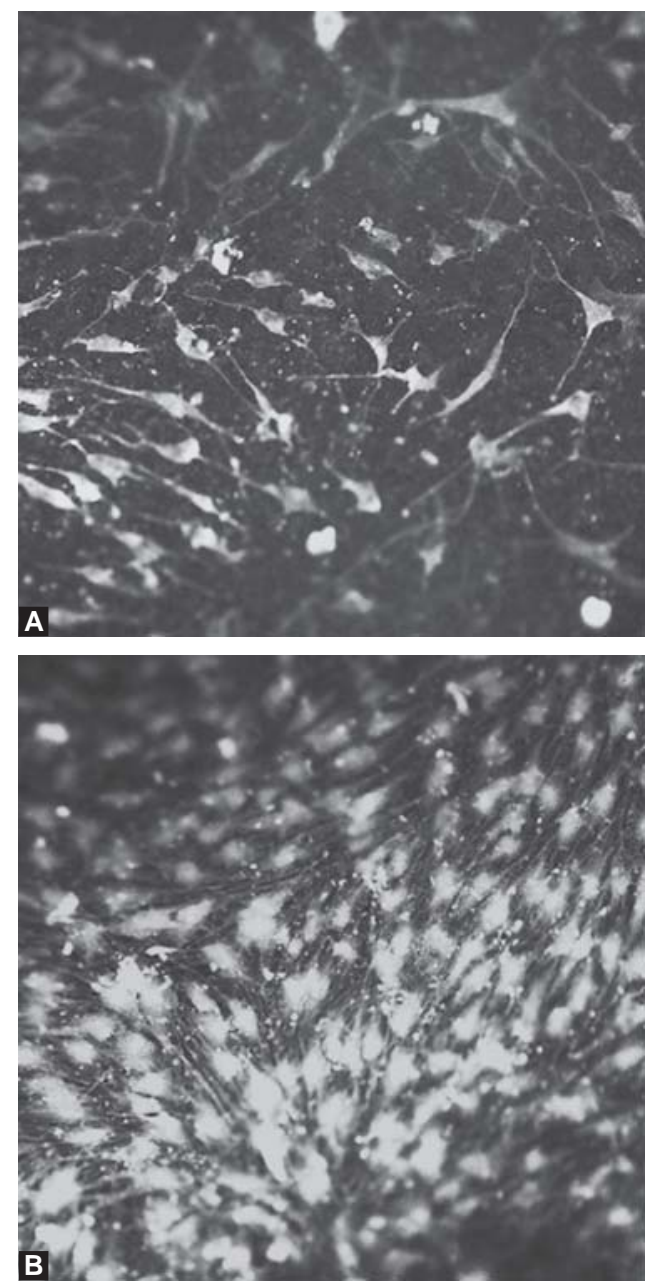

Figs 5A and B: Titanium surfaces at confocal microscope (A) machined, (B) blasted

\section{DISCUSSION AND CONCLUSION}

In 1990, Williams ${ }^{10}$ stated that ultrastructural, microstructural and macro-levels of the surface topography are known to influence the behavior of the adjacent tissue.

There are numerous reports that demonstrate that the surface roughness of titanium implants affects the rate of osseointegration and biomechanical fixation. ${ }^{11,12}$

Several reports have shown that both the early fixation and long-term mechanical stability of the prosthesis can be improved by a high roughness profile compared to smooth surfaces. ${ }^{7,13,14}$ The high roughness resulted in mechanical interlocking between the implant surface and bone on growth. However, a major risk with high surface roughness may be an increase in peri-implantitis as well as an increase in ionic leakage. ${ }^{15}$

The main clinical indication for using an implant with a rough surface is the poor quality or volume of the host bone. ${ }^{16}$ In these unfavorable clinical situations, early and high bone-to-implant contact would be beneficial for allowing high levels of loading. ${ }^{17}$ In the cases of insufficient bone quantity or anatomical limitations, short designed implants with a rough surface have demonstrated superior clinical outcomes than smooth surfaces. ${ }^{18,19}$ Numerous studies have shown that surface roughness in this range resulted in greater bone-to-implant contact and higher resistance to torque removal than other types of surface topography. ${ }^{12,14}$ These reports have demonstrated that titanium implants with roughened surfaces have greater contact with bone than titanium implants with smoother surfaces. ${ }^{11,12}$ However, the Cochrane collaboration has not found any clinical evidence demonstrating the superiority of any particular implant surface. ${ }^{20}$

There are a number of surfaces commercially available for dental implants. M ost of these surfaces have proven clinical efficacy (>95\% over 5 years). However, the development of these surfaces has been empirical, requiring numerous in vitro and in vivo tests. M ost of these tests were not standardized, using different surfaces, cell populations or animal models. The exact role of surface chemistry and topography on the early events of the osseointegration of dental implants remain poorly understood. Furthermore, comparative clinical studies with different implant surfaces are rarely performed.

In agreement with most investigations of bone growth on rough and smooth surfaces the results demonstrate superior osteoblasts fixation for blasted implants compared with the smoother as-machined implants.

These observations indicate that after surfaces treatment, adhesion factors, respiratory activity and expression of osteogenic elements regulate osteoblasts growth and differentiation. Further extension of these studies, in combination with trials with different implant materials should result strategic in devising materials and procedures for new and effective regenerative and prosthetic dental treatments.

\section{REFERENCES}

1. Albrektsson $T$, Dahl E, Enbom L, Engevall S, Engquist $B$, Eriksson AR, et al. Osseointegrated oral implants. A Swedish multicenter study of 8139 consecutively inserted Nobel pharma implants. J Periodontol $1988 \mathrm{M}$ ay;59(5):287-96.

2. L ekholm U, van Steenberghe D, Herrmann L, B olender C, Folmer T, Gunne J, etal. Osseointegrated implants in the treatment of partially edentulous jaws: A prospective 5-years multicenter study. Int J Oral M axillofac Implants 1994;9(6):627-35.

3. Koth DL, M CK inney RV, Steflik DE, Davis QB. Clinical and statistical analyses of human clinical trials with single crystal aluminum oxide endosteal dental implants: Five-year results. J Prosthet D ent 1988;60:226-34.

4. M almquist T, Sennerby L. Clinical report on the success of 47 consecutive placed corevent implants followed from 3 months to 4 years. Int J Oral M axillofac Implants 1990;5:53-60. 
5. A vidsson $K$, Bystedt $H$, Frykholm $A$, von $K$ onow $L$, L othigius $E$. A 3-year clinical study of A stra dental implants in the treatment of edentulous mandibles. Int J Oral Maxillofac Implants 1992; 7:321-29.

6. Albrektsson $\mathrm{T}, \mathrm{B}$ rånemark $\mathrm{PI}, \mathrm{H}$ ansson $\mathrm{HA}$, Lindström J. Osseointegrated titanium implants. Requirements for ensuring a long-lasting, direct bone-to-implant anchorage in man. A cta Orthop Scand 1981;52:155-70.

7. Buser D, Schenk RK, Steinemann S, Fiorellini JP, Fox CH, Stich $\mathrm{H}$. Influence of surface characteristics on bone integration of titanium implants. A histomorphometric study in miniature. J Biomed M ater Res 1991;25:889-902.

8. Grassi FR, Ciccolella F, D'A polito G, Papa F, I uso A, Salzo $A E$, et al. Effect of low-level laser irradiation on osteoblast proliferation and bone formation. J B iol Regul Homeost A gents 2011 Oct-Dec;25(4):603-14.

9. Puchtler $\mathrm{H}, \mathrm{M}$ eloan $\mathrm{SN}$. D emonstration of phosphates in calcium deposits: A modification of von K ossa's reaction. Histochemistry 1978:56:177-85.

10. Williams DF. Biocompatibility: Performance in the surgical reconstruction of man. Interdisciplinary Science Reviews 1990;15(1):20-33.

11. Cochran DL, Schenk RK, L ussi A, Higginbottom FL, B user D. $B$ one response to unloaded and loaded titanium implants with a sandblasted and acid-etched surface: A histometric study in the canine mandible. J Biomed M ater Res 1998;40:1-11.

12. Wennerberg A, Hallgren C, Johansson C, Danelli S. A histomorphometric evaluation of screw-shaped implants each prepared with two surface roughnesses. Clin Oral Implants Res 1998;9:11-19.

13. Gotfredsen $K$, W ennerberg $A$, J ohansson $C$, Skovgaard $L T$, Hjorting-H ansen $\mathrm{E}$. A nchorage of $\mathrm{TiO}_{2}$-blasted, $\mathrm{HA}$-coated, and machined implants: A $n$ experimental study with rabbits. J Biomed M ater Res 1995;29:1223-31.

14. Wennerberg A, Albrektsson T, Albrektsson B, K rol JJ. Histomorphometric and removal torque study of screw-shaped titanium implants with three different surface topographies. Clin Oral Implant Res 1996;6:24-30.

15. Becker W, Becker BE, Ricci A, B ahat 0 , Rosenberg E, Rose $L F$, et al. A prospective multicenter clinical trial comparing onestage plasma-sprayed solid-screw fixtures. Clin Implant Dent Relat Res 2000;2:159-65.

16. A lbrektsson $T, W$ ennerberg $A$. The impact of oral implantspast and future, 1966-2042. J Can Dent A ssoc 2005;71:327.

17. Hansson $S, N$ orton $M$. The relation between surface roughness and interfacial shear strength for bone-anchored implants. A mathematical model. J Biomech 1999;32:829-36.

18. Testori T, Wiseman L, Woolfe S, Porter S. A prospective multicenter clinical study of the Osseotite implant: Four-year interim report. IntJ Oral M axill ofac I mplants 2001;16:193-200.

19. Conner K, Sabatini R, M ealey B, Takacks V, M ills M, Cochran $D$. Guided bone regeneration around titanium plasma-sprayed, acid-etched and hydroxyapatite-coated implants in the canine model. J Periodontol 2003;74:658-68.

20. Esposito $M$, Coulthard $P$, Thomsen $P$, Worthington HV. Interventions for replacing missing teeth: Different types of dental implants. Cochrane Database Syst Rev 2005;25:CD 003815.

\section{ABOUT THE AUTHORS}

\section{Andrea Ballini}

Investigator, Head of Biotech Laboratory, Department of Dental Sciences and Surgery, U niversity of B ari 'A Ido M oro', Italy

\section{Apollonia Desiate}

Associate Professor, Department of Dental Sciences and Surgery, University of Bari 'A ldo M oro', Italy

\section{Stefania Cantore (Corresponding Author)}

Lecturer, D epartment of D ental Sciences and Surgery, University of Bari 'A Ido M oro', I taly, e-mail: stefykant@libero.it 